PROCEEDINGS OF THE

AMERICAN MATHEMATICAL SOCIETY

Volume 140, Number 8, August 2012, Pages 2647-265

S 0002-9939(2011)11285-1

Article electronically published on December 21, 2011

\title{
RELATIONS BETWEEN TWISTED DERIVATIONS AND TWISTED CYCLIC HOMOLOGY
}

\author{
JACK M. SHAPIRO
}

(Communicated by Birge Huisgen-Zimmermann)

\begin{abstract}
For a given endomorphism on a unitary $k$-algebra, $A$, with $k$ in the center of $A$, there are definitions of twisted cyclic and Hochschild homology. This paper will show that the method used to define them can be used to define twisted de Rham homology. The main result is that twisted de Rham homology can be thought of as the kernel of the Connes map from twisted cyclic homology to twisted Hochschild homology.
\end{abstract}

For a noncommutative algebra $A$, Connes [1] and Karoubi 4] define the module of $n$-forms by taking the iterated tensor product of the bimodule of 1 -forms. Karoubi in 4, Chapter 2] defines noncommutative de Rham homology. For the case where $A$ is an associative unitary algebra over a commutative ring, $k$, containing $\mathbb{Q}$, he shows that the reduced noncommutative de Rham homology is isomorphic to the kernel of the Connes map $B$ from $\bar{H} C_{n}(A)$, reduced cyclic homology, to $\bar{H} H_{n+1}(A)$, reduced Hochschild homology. The bimodule of 1-forms used comes from the derivation $d: A \rightarrow A \otimes_{k} A$, with $d(a)=1 \otimes a-a \otimes 1$. In this paper we will generalize that result to the case of twisted cyclic homology based on a given $k$-algebra endomorphism, $h: A \rightarrow A$. Here the bimodule of 1 -forms will come from the twisted derivation, $d^{h}: A \rightarrow A \otimes_{k} A$, with $d^{h}(a)=1 \otimes a-h(a) \otimes 1$. If $h=i d$, then we have $d^{h}=d$. A twisted derivation is any $k$-linear map, $d^{h}$, from $A$ to an $A$-bimodule such that $d^{h}(a b)=d^{h}(a) \cdot b+h(a) \cdot d^{h}(b)$. The definitions for twisted Hochschild homology and twisted cyclic homology are given in [3]. As pointed out there, in the twisted case $C_{*}(A)$ itself is a paracyclic object, and we need to take an appropiate quotient of $C_{*}(A)$ to obtain a cyclic object. It is quite interesting that even though the intrinsic definitions of the $h$-twisted theories differ considerably from the classical nontwisted theories, still there is an appropriate extension of de Rahm homology to the twisted case and an extension of the maps between all the twisted theories that give us a generalization of [4, Theorem 2.15].

Theorem. Suppose $A$ is a unitary k-algebra with $\mathbb{Q} \subset k$. If $h$ is a $k$-algebra endomorphism then the reduced twisted de Rham homology and the reduced twisted cyclic homology are related by the exact sequence

$$
0 \rightarrow \bar{H} D R_{n}^{h}(A) \rightarrow \bar{H} C_{n}^{h, \lambda}(A) \rightarrow \bar{H} H_{n+1}^{h}(A) .
$$

$\bar{H} H_{*}^{h}(A)$ is the reduced twisted Hochschild homology and $\bar{H} C_{*}^{h, \lambda}(A)$ is the reduced twisted cyclic homology based on the Connes complex $\left\{\bar{C}_{n}^{h, \lambda}(A), b^{h}\right\}$. Details of the Connes complex together with the definition of $\bar{H} D R_{n}^{h}(A)$ will be given

Received by the editors March 11, 2009 and, in revised form, March 15, 2011.

2010 Mathematics Subject Classification. Primary 16E40; Secondary 16T20.

(C)2011 American Mathematical Society Reverts to public domain 28 years from publication 
in section 2. As an application of the theorem, we will show how some parts of $\bar{H} C_{*}^{h}\left(\mathcal{A}\left(S L_{q}(2)\right)\right)$, computed in [4, $\left.\S 5.2\right]$, come from twisted de Rham homology. Since their definitions come from the study of quantum groups, they are given with respect to a $k$-algebra automorphism, but they work equally well for any $k$-algebra endomorphism.

\section{Twisted DERivations}

Suppose we have an associative unitary algebra $A$ over a ring $k$ with the map $r \rightarrow r \cdot 1, r \in k$, an isomorphism from $k$ to a $k$-subalgebra lying in the center of $A$. $\bar{A}$ represents $A / k$ and $A \otimes A$ will stand for $A \otimes_{k} A$.

Definition 1. Given a $k$-algebra endomorphism $h$ on $A$, an $h$-twisted derivation is a $k$-linear map $d^{h}: A \rightarrow M$, where $M$ is an $A$-bimodule, satisfying the relation that $d^{h}(a b)=d^{h}(a) b+h(a) d^{h}(b)$.

Example. Let $I^{h}$ be the sub- $A$-bimodule of $A \otimes A$ generated by the terms $\{a \otimes$ $b-a h(b) \otimes 1: a, b \in A\}$. If $d^{h} b$ represents the element $1 \otimes b-h(b) \otimes 1$, then every element in $I^{h}$ can be written as a linear combination of terms of the form $a d^{h} b$. Multiplication on the left is straightforward, while multiplication on the right is given by $a d^{h} b \cdot c=a d^{h}(b c)-a h(b) d^{h} c$. This makes the map $d^{h}: A \rightarrow I^{h}$, given by $d^{h}(a)=1 \otimes a-h(a) \otimes 1$, an $h$-twisted derivation, as a left $A$-module $I^{h}$ is isomorphic to $A \otimes \bar{A}$ under the isomorphism sending $a \otimes \bar{b}$ to $a d^{h} b$. This is proved in [7, Lemma 3.1]. The statement made there is for an automorphism but the proof goes through equally well for any endomorphism. We can also view $I^{h}$ as the kernel of a multiplication map, given by $\mu^{h}(a \otimes b)=a \cdot h(b)$. If $\sum_{i=1}^{n} a_{i} \otimes b_{i}$ is in the kernel of $\mu^{h}$, then we have $\sum_{i=1}^{n} a_{i} \cdot h\left(b_{i}\right)=0$. If so, we can rewrite $\sum_{i=1}^{n} a_{i} \otimes b_{i}$ as $\sum_{i=1}^{n} a_{i} \otimes b_{i}-a_{i} h\left(b_{i}\right) \otimes 1 \in I^{h}$. That $I^{h}$ is in the kernel of $\mu^{h}$ is easy to see.

In 7 it is proved that $\left(I^{h}\right)^{\otimes_{A}^{n}} \simeq \bar{C}_{n}(A) \equiv A \otimes(\bar{A})^{\otimes^{n}}$, as left $A$-modules. The map is given by $a_{0} d^{h}\left(a_{1}\right) \cdots d^{h}\left(a_{n}\right) \rightarrow a_{0} \otimes \bar{a}_{1} \otimes \cdots \otimes \bar{a}_{n} \equiv\left(a_{0}, \bar{a}_{1}, \cdots, \bar{a}_{n}\right)$.

\section{Twisted Hochschild AND TWISTED CYCLIC HOMOLOGY}

The definitions of twisted Hochschild and twisted cyclic homology in [3] are based on the definition of [5] for twisted cyclic cohomology. Given a $k$-algebra automorphism $h$ of a $k$-algebra $A$, they define $C_{n}^{h}(A)$ as the quotient of $C_{n}(A)$ by the image of $(1-T): C_{n}(A) \rightarrow C_{n}(A)$, where $T\left(a_{0}, a_{1}, \cdots, a_{n}\right)=\left(h\left(a_{0}\right), h\left(a_{1}\right), \cdots, h\left(a_{n}\right)\right)$. As mentioned in the introduction, the quotient is needed to obtain a cyclic object. Next they define $b^{h}=\sum_{i=0}^{n}(-1)^{i} b_{i}$, where for $0 \leq i \leq n-1, b_{i}$ is the same as $d_{i}$ in [6], and $b_{n}$ is given by $b_{n}\left(a_{0}, a_{1}, \cdots, a_{n}\right)=\left(h\left(a_{n}\right) a_{0}, a_{1}, \cdots, a_{n-1}\right)$. Using the complex $\left(C_{n}^{h}(A), b^{h}\right)$, they define twisted Hochschild homology, which they denote as $H H_{n}^{h}(A)$. To get twisted cyclic homology, they define $B^{h}: C_{n}^{h}(A) \rightarrow C_{n+1}^{h}(A)$, and from that they get a double complex similar to [6, §2.5.10]. As in the nontwisted case the formula for $B^{h}$ is simpler after normalization. That is, we take $\bar{C}_{n}^{h}(A)$ to be the quotient of $C_{n}^{h}(A)$ by the $k$-submodule generated by all terms $\left(a_{0}, a_{1}, \cdots, a_{n}\right)$ such that at least one of the $a_{i}=1$, for some $1 \leq i \leq n$. In the normalized case, $B^{h}$ can be given by the formula

$$
B^{h}\left(a_{0}, \bar{a}_{1}, \cdots, \bar{a}_{n}\right)=\sum_{i=0}^{n}(-1)^{n i}\left(1, h\left(\bar{a}_{i}\right), \cdots, h\left(\bar{a}_{n}\right), \bar{a}_{0}, \cdots, \bar{a}_{i-1}\right) .
$$


From this double complex they define twisted cyclic homology. As mentioned in the introduction, all of the above goes through for any $k$-algebra endomorphism.

The same "modding out" by $(1-T)$ can be done on the differential, $d^{h}: \bar{C}_{n}^{h}(A) \rightarrow$ $\bar{C}_{n+1}^{h}(A)$, given by $d^{h}\left(a_{0}, \bar{a}_{1}, \cdots, \bar{a}_{n}\right)=\left(1, \bar{a}_{0}, \bar{a}_{1}, \cdots, \bar{a}_{n}\right)$. This follows from the fact that

$$
\begin{aligned}
d^{h}\left(h\left(a_{0}\right), h\left(\bar{a}_{1}\right), \cdots, h\left(\bar{a}_{n}\right)\right) & =\left(1, h\left(\bar{a}_{0}\right), h\left(\bar{a}_{1}\right), \cdots, h\left(\bar{a}_{n}\right)\right) \\
& =\left(1, \bar{a}_{0}, \bar{a}_{1}, \cdots, \bar{a}_{n}\right)=d^{h}\left(a_{0}, \bar{a}_{1}, \cdots, \bar{a}_{n}\right) .
\end{aligned}
$$

The maps $d^{h}$ and $b^{h}$ as maps on $\bar{C}_{n}^{h}(A)$ are related by the formula $b^{h} \circ d^{h}+d^{h} \circ b^{h}=$ $(1-\kappa)$ [7, Lemma 3.2]. $\kappa$ is the Karoubi operator given by the formula $\kappa\left(a_{0}, \bar{a}_{1}, \cdots, \bar{a}_{n}\right)=(-1)^{n}\left[\left(h\left(a_{n}\right), \bar{a}_{0}, \bar{a}_{1}, \cdots, \bar{a}_{n-1}\right)-\left(1, h\left(\bar{a}_{n}\right) \cdot \bar{a}_{0}, \bar{a}_{1}, \cdots, \bar{a}_{n-1}\right)\right]$.

For $h=i d$, we have $\kappa=\sigma$, the endomorphism appearing in [6, §2.6.8]. There the formula is given as $b^{h} \circ d^{h}+d^{h} \circ b^{h}=(1-\sigma)$. From the above it follows that $\kappa$ commutes both with $b^{h}$ and $d^{h}$.

Let $\bar{C}_{n}^{h}(A)^{\kappa}$ denote $\frac{\bar{C}_{n}^{h}(A)}{\operatorname{Im}(1-\kappa)}$, and from this define $\bar{C}_{n}^{h}(A)_{a b}$ as

$$
\frac{\bar{C}_{n}^{h}(A)^{\kappa}}{\operatorname{Im}\left(b^{h}\right)}=\frac{\bar{C}_{n}^{h}(A)}{\operatorname{Im}(1-\kappa)+\operatorname{Im}\left(b^{h}\right)} .
$$

For $h=i d$, it is shown in [6, proof of Lemma 2.6.8] that $\bar{C}_{n}(A)_{a b}$ is the abelianization of $\bar{C}_{n}(A)$, hence the choice of notation. The map $d^{h}$ can be used to give us the chain complexes, $\left(\bar{C}_{n}^{h}(A)^{\kappa}, d^{h}\right)$ and $\left(\bar{C}_{n}^{h}(A)_{a b}, d^{h}\right)$.

The following is a generalization of noncommutative de Rham homology; see [6].

Definition 2. Given a $k$-algebra endomorphism, $h$, of $A$, the twisted de Rham homology of $A$ is

which we will denote as $H D R_{n}^{h}(A)$.

$$
H_{n}\left(\bar{C}_{*}^{h}(A)_{a b}, d^{h}\right),
$$

At the end of the introduction it was noted that $\bar{H} C_{*}^{h, \lambda}(A)$ represents the reduced twisted cyclic homology coming from a Connes complex. Here are the details. We get the Connes complex from quotients of $C_{n}^{h}(A)$ by the image of the map, $(1-t): C_{n}^{h}(A) \rightarrow C_{n}^{h}(A)$, where $t$ is given by $t\left(a_{0}, a_{1}, \cdots, a_{n}\right)=$ $(-1)^{n}\left(h\left(a_{n}\right), a_{0}, \cdots, a_{n-1}\right)$. The quotient is denoted as $C_{n}^{h, \lambda}(A)$. It turns out that $b^{h}$ is well defined on $C_{n}^{h, \lambda}(A)$ and yields a chain complex $\left(C_{n}^{h, \lambda}(A), b^{h}\right)$. To get reduction, we mod out by the $k$-submodule generated by all terms of the form $\left(a_{0}, a_{1}, \cdots, a_{n}\right)$, where at least one of the $a_{i}=1, i \geq 0$, and denote the quotient as $\bar{C}_{n}^{h, \lambda}(A)$. The homology of the complex $\left(\bar{C}_{n}^{h, \lambda}(A), b^{h}\right)$ is denoted as $\bar{H} C_{*}^{h, \lambda}(A)$. When $\operatorname{ch}(k)=0$ this homology is the same as the twisted cyclic homology we get from the double complex [2], and that is exactly the case we are dealing with in the main theorem.

Another way of getting reduction on $C_{n}^{h, \lambda}(A)$ is to mod out by $\operatorname{Im}\left(d^{h}\right)$. That is,

$$
\bar{C}_{n}^{h, \lambda}(A)=\frac{C_{n}^{h, \lambda}(A)}{\operatorname{Im}\left(d^{h}\right)}=\frac{\bar{C}_{n}^{h}(A)}{\operatorname{Im}(1-t)+\operatorname{Im}\left(d^{h}\right)} .
$$

In modding out by $\operatorname{Im}(1-t)$ and $\operatorname{Im}\left(d^{h}\right)$, we get that every $\left(a_{0}, a_{1}, \cdots, a_{n}\right)$ with any $a_{i}=1, i \geq 0$, is modded out. Conversely, when you make reduction, as above, you have already modded out everything in $\operatorname{Im}\left(d^{h}\right)$. With this in mind we have an easy proof of the following lemma. 
Lemma 1. $\frac{\bar{C}_{n}^{h}(A)_{a b}}{\operatorname{Im}\left(d^{h}\right)} \simeq \frac{\bar{C}_{n}^{h, \lambda}(A)}{\operatorname{Im}\left(b^{h}\right)}$.

Proof. In $\frac{\bar{C}_{n}^{h}(A)}{\operatorname{Im}\left(d^{h}\right)}$ we have $\kappa=t$. Hence both are isomorphic to

$$
\frac{\bar{C}_{n}^{h}(A)}{\operatorname{Im}(1-t)+\operatorname{Im}\left(b^{h}\right)+\operatorname{Im}\left(d^{h}\right)} .
$$

Using the method in [6, §1.4.2], applied to $\bar{C}_{*}^{h}(A)$, we get the reduced twisted Hochschild homology $\bar{H} H_{n}^{h}(A)$. A similar procedure applied to $\bar{C}_{*}^{h}(A)_{a b}$ yields the reduced twisted de Rham homology, $\bar{H} D R_{n}^{h}(A)$, which is isomorphic to $H D R_{n}^{h}(A)$ for $n>0$.

Lemma 2. If $k$ contains $\mathbb{Q}$, then we can view $\bar{H} H_{n}^{h}(A)$ as a submodule of $\bar{C}_{n}^{h}(A)_{a b}$.

Proof. To start with we have for $n \geq 1$

$$
\bar{H} H_{n}^{h}(A)=\frac{\operatorname{ker}\left(b^{h}\right)}{\operatorname{Im}\left(b^{h}\right)} \leq \frac{\bar{C}_{n}^{h}(A)}{\operatorname{Im}\left(b^{h}\right)} .
$$

Using the fact that $\kappa$ has order $\mathrm{n}$ in $\frac{\bar{C}_{n}^{h}(A)}{\operatorname{Im}\left(b^{h}\right)}$ and the formula $b^{h} \circ d^{h}+d^{h} \circ b^{h}=(1-\kappa)$ [7, it follows that $\bar{H} H_{n}^{h}(A)$ is contained in the module of invariants, $\left(\frac{\bar{C}_{n}^{h}(A)}{\operatorname{Im}\left(b^{h}\right)}\right)^{\kappa}$. Since $k$ contains $\mathbb{Q}$, we have that $\left(\frac{\bar{C}_{n}^{h}(A)}{\operatorname{Im}\left(b^{h}\right)}\right)^{\kappa} \simeq \frac{\bar{C}_{n}^{h}(A)}{\operatorname{Im}(1-\kappa)+\operatorname{Im}\left(b^{h}\right)}=\bar{C}_{n}^{h}(A)_{a b}$. For $n=0,(1-\kappa)$ is the zero map on $\bar{C}_{0}^{h}(A)$, and we get that $\bar{H} H_{0}^{h}(A)=\bar{C}_{0}^{h}(A)_{a b}$.

Using the formulas in [7, $B^{h}=\sum_{j=0}^{n} \kappa^{j} \circ d^{h}$ and $\kappa^{n+1} \circ d^{h}=d^{h}$, it follows that $\kappa \circ B^{h}=B^{h} \circ \kappa=B^{h}$. This gives us a well defined map, $B^{h}: \bar{C}_{n}^{h}(A)_{a b} \rightarrow \frac{\bar{C}_{n+1}^{h}(A)}{\operatorname{Im}\left(b^{h}\right)}$. Using the above formula for $B^{h}$ and the fact that in $\bar{C}_{n}^{h}(A)_{a b}$ we have $\kappa=$ id, we get the twisted version of [6, Lemma 2.6.9].

Lemma 3. There is a commutative diagram:

$$
\begin{array}{ccc}
\bar{C}_{n}^{h}(A) & \stackrel{B^{h}}{\longrightarrow} & \bar{C}_{n+1}^{h}(A) \\
\downarrow \pi & & \downarrow \pi \\
\bar{C}_{n}^{h}(A)_{a b} & \stackrel{(n+1) d^{h}}{\longrightarrow} & \frac{\bar{C}_{n+1}^{h}(A)}{\operatorname{Im}\left(b^{h}\right)} .
\end{array}
$$

We now have all the facts needed to generalize Karoubi's theorem.

Theorem. Suppose $A$ is a unitary $k$-algebra with $\mathbb{Q} \subset k$. If $h$ is a $k$-algebra endomorphism, then twisted de Rham homology and twisted cyclic homology are related by the following exact sequence:

$$
0 \rightarrow \bar{H} D R_{n}^{h}(A) \rightarrow \bar{H} C_{n}^{h, \lambda}(A) \rightarrow \bar{H} H_{n+1}^{h}(A) .
$$

That is, we can think of $\bar{H} D R_{n}^{h}(A)$ as the kernel of the Connes map, $B^{h}$ : $\bar{H} C_{n}^{h, \lambda}(A) \rightarrow \bar{H} H_{n+1}^{h}(A)$. As stated above, with $\operatorname{ch}(k)=0$ we have $\bar{H} C_{n}^{h, \lambda}(A)=$ $\bar{H} C_{n}^{h}(A)$, reduced twisted cyclic homology coming from the double complex. 
Proof. Using the diagram below we can view the three terms appearing in the exact sequence as the kernels of the three vertical arrows appearing in the diagram. Keep in mind that $\gamma$ is an isomorphism by Lemma 1 and that $B^{h} \circ \gamma=(n+1) d^{h}$ by Lemma 3. The rest is just a generalization of [4, 2.14, 2.15]:

$$
\begin{array}{cccccc}
\frac{\bar{C}_{n}^{h}(A)_{a b}}{I m\left(d^{h}\right)} & \stackrel{\gamma}{\rightarrow} & \frac{\bar{C}_{n}^{h, \lambda}(A)}{I m\left(b^{h}\right)} & \stackrel{B^{h}}{\rightarrow} & \frac{\bar{C}_{n+1}^{h}(A)}{\operatorname{Im}\left(b^{h}\right)} \\
\downarrow d^{h} & & \downarrow b^{h} & & \downarrow b^{h} \\
\bar{C}_{n+1}^{h}(A)_{a b} & & & \bar{C}_{n-1}^{h, \lambda}(A) & \stackrel{B^{h}}{\rightarrow} & \bar{C}_{n}^{h}(A) .
\end{array}
$$

Application. In section 5 of 3 calculations are made for the twisted cyclic homology of $A=\mathcal{A}\left(S L_{q}(2)\right)$, using the spectral sequence of the Connes mixed $\left(b^{h}, B^{h}\right)$-bicomplex. Through early stabilization they derive some of the twisted cyclic homology from the kernel of the maps, $\left(B^{h}\right): \bar{H} H_{n}^{h}(A) \rightarrow \bar{H} H_{n+1}^{h}(A)$, for $n=0,1,2$. Using Lemma 2, we have an injection, $\bar{H} H_{n}^{h}(A) \hookrightarrow \bar{C}_{n}^{h}(A)_{a b}$, which can be followed with $\bar{C}_{n}^{h}(A)_{a b} \Rightarrow \frac{\bar{C}_{n}^{h}(A)_{a b}}{\operatorname{Im}\left(d^{h}\right)}$. Next, composing with $B^{h} \circ \gamma$, we induce the map $\left(B^{h}\right): \bar{H} H_{n}^{h}(A) \rightarrow \bar{H} H_{n+1}^{h}(A)$, since by the properties of the bicomplex, $B^{h}$ takes the $\operatorname{ker}\left(b^{h}\right)$ to the $\operatorname{ker}\left(b^{h}\right)$, and all the other maps are induced by the identity on $\bar{C}_{n}^{h}(A)$. The fact that $B^{h} \circ \gamma=(n+1) d^{h}$, means that elements of $\operatorname{ker}\left(B^{h}: \bar{H} H_{n}^{h}(A) \rightarrow \bar{H} H_{n+1}^{h}(A)\right)$, map to $\bar{H} D R_{n}^{h}(A)$, under the maps $\bar{H} H_{n}^{h}(A) \hookrightarrow \bar{C}_{n}^{h}(A)_{a b} \Rightarrow \frac{\bar{C}_{n}^{h}(A)_{a b}}{\operatorname{Im}\left(d^{h}\right)}$. Hence those parts of $\bar{H} C_{n}^{h}(A)$ coming from $\operatorname{ker}\left(B^{h}: \bar{H} H_{n}^{h}(A) \rightarrow \bar{H} H_{n+1}^{h}(A)\right)$ are elements of $\bar{H} D R_{n}^{h}(A)$, sitting inside $\bar{H} C_{n}^{h}(A)$. That is, they come from twisted de Rham homology.

\section{REFERENCES}

[1] Connes, A., Noncommutative differential geometry, Publ. Math. IHES, 62 (1985), 257360. MR.823176 (87i:58162)

[2] Hadfield, T., Twisted cyclic homology of all Podles quantum spheres, Journal of Geometry and Physics, 57(2) (2007), 339-351. MR2271192 (2007m:58008)

[3] Hadfield, T., Krähmer, U., Twisted homology of quantum SL(2), K-theory, 34(4) (2005), 327-360. MR2242563 (2007j:58009)

[4] Karoubi, M., Homologie cyclique, Astérisque, vol. 149 (1987), Société Mathématique de France. MR.913964 (89c:18019)

[5] Kustermans, J., Murphy, G.J., Tuset, L., Differential calculi over quantum and twisted cyclic cocycles, Journal of Geometry and Physics, 44(4) (2003), 570-594. MR.1943179

[6] Loday, J.-L., Cyclic homology, second edition, Springer, Grundlehren der mathematischen Wissenschaften, volume 301 (1997), Springer-Verlag, 1998. MR:1600246|(98h:16014)

[7] Redondo, M.J., Solotar, A., Paracyclic complexes arising from $\alpha$-derivations, Proceedings of the $4^{\text {th }}$ Dr. Antonio A. R. Monteiro Congress on Mathematics (Bahía Blanca), 1997, MR1619239(99i:16019)

Department of Mathematics, Washington University, Saint Louis, Missouri 63130

E-mail address: jshapiro@math.wustl.edu 